\title{
Efeito das concentrações de farinha de \\ cascas de banana e de sacarose nas \\ características físicas e químicas de bolos
}

\section{Effect of concentrations of banana peel flour and sucrose on physical and chemical characteristics in cakes}

\section{Lucilene Marques da Costa Oliveira1, Maria de Jesus Lima Pereira1, Dyego da Costa Santos ${ }^{1 *}$ (1), Daniela Dantas de Farias Leite ${ }^{2}$, Thalis Leandro Bezerra Lima ${ }^{2}$, Josivanda Palmeira Gomes ${ }^{2}$}

${ }^{1}$ Instituto Federal de Educação, Ciência e Tecnologia do Acre (IFAC), Departamento de Tecnologia em Agroindústria, Xapuri/AC - Brasil

${ }^{2}$ Universidade Federal de Campina Grande (UFCG), Unidade Acadêmica de Engenharia Agrícola, Campina Grande/PB - Brasil

*Corresponding Author: Dyego da Costa Santos, Instituto Federal de Educação, Ciência e Tecnologia do Acre (IFAC), Departamento de Tecnologia em Agroindústria, Rua Coronel Brandão, 1622, Centro, CEP: 69930-000, Xapuri/AC - Brasil, e-mail: dyego.csantos@gmail.com

Cite as: Oliveira, L. M. C., Pereira, M. J. L., Santos, D. C., Leite, D. D. F., Lima, T. L. B., \& Gomes, J. P. (2020). Effect of concentrations of banana peel flour and sucrose on physical and chemical characteristics in cakes.

Brazilian Journal of Food Technology, 23, e2019314. https://doi.org/10.1590/1981-6723.31419

\begin{abstract}
Resumo
A banana é uma das frutas mais consumidas no mundo por causa de suas características sensoriais agradáveis e composição nutricional. Depois do consumo da polpa, geram-se grandes quantidades de cascas que, após desidratação e transformação em farinha, podem ser inseridas em formulações de bolos. Nesse sentido, objetivou-se avaliar a influência das concentrações de farinha de cascas de banana D'Angola e de açúcar nas características físicas e químicas de bolos. Estes foram processados mediante planejamento fatorial experimental $2^{2} \mathrm{com}$ três repetições no ponto central, resultando em sete experimentos. Preparou-se uma mistura composta de farinha de trigo, fermento químico, manteiga, leite integral e ovos, com posterior acréscimo de diferentes níveis de farinha de cascas de banana e de açúcar cristal. Após cocção de $28 \mathrm{~min}$ a $200^{\circ} \mathrm{C}$ e resfriamento, os bolos foram submetidos a análises químicas e físicas. Observou-se que os modelos matemáticos ajustados aos dados experimentais de sólidos solúveis totais (SST), acidez total titulável (ATT), pH e relação SST/ATT foram significativos e preditivos, com coeficientes de correlação superiores a 0,98 e relação $F_{\text {calculado }} / F_{\text {tabelado }}$ superior a 4,0. Os SST sofreram influência significativa apenas do açúcar, que também foi a variável independente que mais afetou a relação SST/ATT. Em contrapartida, a de farinha de cascas de banana apresentou maior influência sobre os resultados de ATT e de pH.
\end{abstract}

Palavras-chave: Musa spp.; Aproveitamento agroindustrial; Agregação de valor; Produto de panificação; Controle de qualidade; Processo otimizado.

\section{Abstract}

Banana is one of the most consumed fruits in the world due to its pleasant sensory characteristics and nutritional composition. After consumption of the pulp, large amounts of peels are generated which, after dehydration and 
flour transformation, can be inserted into cake formulations. In this sense, the objective of this work was to evaluate the influence of D'Angola banana peel flour and sugar concentrations on the physical and chemical characteristics of cakes. These were processed by experimental factorial design $2^{2}$ with 3 repetitions at the central point, resulting in 7 experiments. A mixture composed of wheat flour, yeast, butter, whole milk and eggs was prepared, with subsequent addition of different levels of banana peel flour and crystal sugar. After cooking for 28 min at $200{ }^{\circ} \mathrm{C}$ and cooling, the cakes were subjected to chemical and physical analysis. The mathematical models adjusted to the experimental data of SST, ATT, pH and SST/ATT ratio were observed to be significant and predictive, with correlation coefficients greater than 0.98 and $F_{\text {calculated }} / F_{\text {tabulated }}$ ratio greater than 4.0. TSS was significantly influenced only by sugar, which was also the independent variable that most affected the TSS/TTA ratio. On the other hand, the banana peel flour factor had a major influence on the ATT and $\mathrm{pH}$ results.

Keywords: Musa spp.; Agro-industrial use; Adding value; Bakery product; Quality control; Formulations process optimized.

\section{Introdução}

A banana, pertencente à família Musaceae, incluindo uma série de híbridos do gênero Musa (Vu et al., 2018), é cultivada nas regiões tropicais e subtropicais de todo o mundo, constituindo-se em alimento básico de muitas populações (Ghag \& Ganapathi, 2019) e em fonte de renda para as pessoas que vivem nas áreas de sua ocorrência (Zhang et al., 2019). É rica em carboidratos, minerais como o potássio e o fósforo (Zhang et al., 2019) e compostos bioativos importantes, por exemplo, compostos fenólicos, flavonoides, carotenoides, aminas biogênicas, esteróis e compostos antimicrobianos, fazendo dessa fruta um alimento funcional (Ghag \& Ganapathi, 2019).

É a segunda fruta mais consumida do planeta (Handique et al., 2019), sendo a Índia o maior produtor, com $1 / 3$ do total da produção mundial (Ghag \& Ganapathi, 2019). O Brasil é o quinto maior produtor (Rocha et al., 2018), com uma produção em 2017 de 6,735 milhões de toneladas em uma área de 468,754 mil hectares (Instituto Brasileiro de Geografia e Estatística, 2019). Atualmente, a safra brasileira é baseada em cultivares do subgrupo Prata, com destaque para o clone Prata Anã (AAB), Gorutuba e Catarina (Rocha et al., 2018), sendo que no Acre as cultivares predominantes são Prata Comum, D'Angola (conhecida regionalmente como banana-comprida), Maçã e Pacovan, além das cultivares que foram recomendadas pela Embrapa Acre: Thap Maeo, Maravilha, Preciosa, Pacovan Ken e Japira (Sá et al., 2016).

Em comparação com outras frutas tropicais, o consumo de banana é elevado principalmente por causa de sua versatilidade no uso, pois ela pode ser consumida in natura e processada nas formas frita, cozida (Borges et al., 2019), assada e incorporada em vários produtos. Considerando-se que, após o consumo e a industrialização da fruta, gera-se uma grande quantidade de cascas, que correspondem a cerca de 35 a $40 \%$ da massa do produto (Khoozani et al., 2019), estima-se que tenham sido gerados no Brasil, apenas no ano de 2017, cerca de 2,357 a 2,694 milhões de toneladas de cascas.

Até pouco tempo atrás, as cascas de banana não tinham aplicações úteis e eram descartadas como resíduos, gerando enormes quantidades de materiais orgânicos a serem manejadas (Khoozani et al., 2019) ou utilizadas como fertilizante orgânico ou ração animal. Entretanto, estudos evidenciaram que esse subproduto contém altos níveis de fibra dietética e fenólicos. Além disso, demonstrou-se que a casca da banana apresenta capacidade antioxidante potente, propriedades antimicrobianas e antibióticas, apresentando-se promissora para diversas aplicações industriais (Vu et al., 2018). Assim, as cascas de banana poderiam ser processadas na forma de farinhas de elevado valor nutricional e bioativo, pois são mais facilmente armazenadas para outros usos (Rebello et al., 2014) e incorporadas em diversos outros produtos.

De acordo com Khoozani et al. (2019), a utilização de subprodutos de frutas, especialmente da banana, tornou-se uma tendência tardia, e muitos estudos recentes estão sendo desenvolvidos para avaliar seus efeitos nas propriedades de novos produtos alimentícios, tais como barras de cereais (Carvalho \& Conti-Silva, 2018), 
macarrões (Castelo-Branco et al., 2017), pães (Eshak, 2016), geleias (Dias et al., 2011), entre outros. Ainda que a casca da banana tenha sido utilizada na confecção de uma grande variedade de produtos, percebe-se que a maioria dos estudos envolvendo o processamento de bolos utiliza a polpa (Silva et al., 2017; Souza et al., 2018; Camargo et al., 2018; Samsudin et al., 2019) em vez das cascas (Sodchit et al., 2013) nos seus processos produtivos. Adicionalmente, são escassas as pesquisas para investigar os efeitos do sinergismo entre cascas de frutas e teores reduzidos de sacarose em bolos.

Uma vez que os ingredientes empregados na elaboração de bolos podem ser substituídos com facilidade (Soares et al., 2018), além da atual preocupação da população em relação à alimentação mais saudável, a farinha de cascas de banana poderia ser utilizada no processamento de bolos comerciais, de modo a melhorar as características funcionais desses produtos, o que, de acordo com Soares et al. (2018), é o segundo gênero mais consumido na panificação. Jeddou et al. (2017) reportaram que o desenvolvimento de alimentos básicos enriquecidos com fibras, como o bolo, é uma contribuição importante para uma oferta mais ampla de produtos alimentícios com efeito benéfico para a saúde. Além disso, a substituição parcial da farinha de trigo pela farinha de cascas de banana pode reduzir o custo de produção dos bolos, uma vez que diminuiria a proporção de um ingrediente relativamente caro, em detrimento de matéria-prima de baixo custo. Essa substituição parcial apresenta benefícios nutricionais e econômicos, todavia se necessita estudar as características físicas e químicas dos novos produtos produzidos, a fim de se conhecer os impactos dessa substituição parcial na qualidade do produto. Nesse sentido, objetivou-se avaliar a influência das concentrações de farinha de cascas de banana D’Angola e de açúcar nas características físicas e químicas de bolos.

\section{Material e métodos}

Utilizou-se de bananas maduras da variedade D’Angola, safra 2019, adquiridas de um pequeno produtor do Polo Agroflorestal de Xapuri, Acre. Para tanto, o estádio de maturação foi determinado segundo a escala de cores para a casca estabelecida por Alves et al. (1999), que classificam como madura a banana que apresente a coloração da casca completamente amarela. A farinha de trigo, o fermento químico, a manteiga, o açúcar cristal (sacarose), o leite UHT integral e os ovos foram provenientes do mercado varejista de Xapuri, Acre.

Os insumos foram transportados adequadamente ao laboratório, onde as bananas foram lavadas em água corrente para a remoção de sujidades das cascas, higienizadas em solução de hipoclorito de sódio com $100 \mathrm{ppm}$ de cloro ativo por $15 \mathrm{~min}$, enxaguadas em água corrente para remoção do agente sanitizante e submetidas à remoção manual das cascas. Estas foram dispostas em bandejas de alumínio e desidratadas em desidratador de alimentos, modelo Pratic Dryer, na temperatura de $60{ }^{\circ} \mathrm{C}$ por $24 \mathrm{~h}$. $\mathrm{Na}$ sequência, elas foram desintegradas em liquidificador, peneiradas em peneiras com malha de 1 mm com o intuito de uniformizar o tamanho dos grânulos da farinha de banana e acondicionada em potes de vidro providos de tampa.

Para o processamento dos bolos à base de farinha das cascas de banana D'Angola e sacarose, utilizou-se de um planejamento experimental fatorial $2^{2}$ com três repetições no ponto central, resultando em sete experimentos, com o intuito de avaliar o efeito de diferentes proporções de farinha de cascas de banana $(5 \%, 10 \%$ e $15 \%)$ e de açúcar cristal $(5 \%, 10 \%$ e $15 \%)$, denominadas variáveis independentes, nas características físicas e químicas dos bolos, denominadas variáveis dependentes. Inicialmente, a farinha de trigo foi pesada e adicionada a 52,59\% de leite, $66,66 \%$ de manteiga e $90,33 \%$ de ovos (todos em relação à massa de farinha de trigo). Os ingredientes foram homogeneizados em batedeira planetária em velocidade média por $8 \mathrm{~min}$, e, posteriormente, adicionaram-se $4,79 \%$ de fermento químico (em relação à massa de farinha de trigo), o qual foi homogeneizado manualmente. Em seguida, a massa de bolo foi dividida em sete partes, as quais foram acrescentadas da farinha de cascas de banana e da sacarose, de acordo com a matriz de planejamento 
disposta na Tabela 1, e homogeneizadas manualmente por $3 \mathrm{~min}$. Salienta-se que as proporções de farinha de cascas de banana e de sacarose foram calculadas em relação à mistura da massa de bolo.

Tabela 1. Matriz de planejamento experimental utilizada no processamento dos bolos.

\begin{tabular}{|c|c|c|c|c|}
\hline \multirow{2}{*}{ Experimento } & \multicolumn{2}{|c|}{ Valores codificados } & \multicolumn{2}{|c|}{ Valores reais } \\
\hline & $\mathbf{x}_{1}$ & $\mathbf{x}_{2}$ & FCB $(\%)$ & Açúcar (\%) \\
\hline B1 & -1 & -1 & 5,0 & 5,0 \\
\hline B2 & +1 & -1 & 15,0 & 5,0 \\
\hline B3 & -1 & +1 & 5,0 & 15,0 \\
\hline B4 & +1 & +1 & 15,0 & 15,0 \\
\hline B5 & 0 & 0 & 10,0 & 10,0 \\
\hline B6 & 0 & 0 & 10,0 & 10,0 \\
\hline B7 & 0 & 0 & 10,0 & 10,0 \\
\hline
\end{tabular}

FCB: Farinha de cascas de banana.

Porções de cerca de $18 \mathrm{~g}$ de massa foram dispostas em formas de alumínio, previamente untadas com manteiga e farinha de trigo e levadas ao forno preaquecido por 20 min e forneadas por 28 min na temperatura de $200{ }^{\circ} \mathrm{C}$. Posteriormente, os bolos, ainda nas formas de alumínio, foram retirados do forno e dispostos em bancada de laboratório, até atingirem temperatura ambiente, e acondicionados em embalagens de polietileno de alta densidade (PEAD). Cada experimento foi processado em quadruplicada, totalizando 28 bolos.

Os bolos de cascas de banana e sacarose foram submetidos a análises físicas e químicas de volumes específicos e aparentes, segundo Pizzinatto \& Campagnolli (1993), massa específica, conforme o método de Zambrano et al. (2005), rendimento, de acordo com Soares et al. (2018), altura com auxílio de paquímetro digital (modelo $316119 \mathrm{MTX}^{\circledR}$ ), teor de umidade, sólidos totais, sólidos solúveis totais (SST), acidez total titulável (ATT) em ácido málico, segundo métodos descritos no Instituto Adolfo Lutz (2008), e relação SST/ATT, quantificada pela relação numérica entre os SST e a ATT.

Os dados da caracterização física e química dos bolos à base de cascas de banana e sacarose foram submetidos à análise estatística por meio da Metodologia da Superfície de Resposta, utilizando-se do programa computacional Statistica, versão 7.0.

\section{Resultados e discussão}

Estão dispostos na Tabela 2 os resultados das análises de rendimento, altura, volume aparente, volume específico e massa específica dos bolos processados com diferentes concentrações de farinha de cascas de banana-comprida e de açúcar. Observou-se que os rendimentos aumentaram com as incorporações de farinha de cascas de banana e de açúcar, com valores compreendidos entre $77,96 \%$ e $82,49 \%$. Isso pode estar relacionado à capacidade de absorção de água pelas fibras presentes na farinha de cascas de banana, que diminuem a água livre presente na massa (Silva et al., 2017) e, consequentemente, reduzem a perda de água durante o forneamento. Além disso, o incremento de açúcar também teria a capacidade de reduzir a água livre, aumentando a quantidade de sólidos da massa. Comportamentos semelhantes foram relados por Jeddou et al. (2017) em bolos formulados com farinha de casca de batata, por Turker et al. (2016) em bolos à base de farinha de cascas de banana-verde e por Rosa et al. (2015) em bolos processados com farinha de alfarroba. 
Tabela 2. Resultados médios das análises de rendimento, altura, volume aparente, volume específico e massa específica dos bolos processados com diferentes concentrações de farinha de cascas de banana e de açúcar.

\begin{tabular}{|c|c|c|c|c|c|}
\hline EXP & Rendimento (\%) & Altura (mm) & $\begin{array}{l}\text { Volume aparente } \\
\qquad\left(\mathrm{cm}^{3}\right)\end{array}$ & $\begin{array}{l}\text { Volume específico } \\
\qquad\left(\mathrm{cm}^{3} / \mathrm{g}\right)\end{array}$ & $\begin{array}{c}\text { Massa específica } \\
\left(\mathbf{g} / \mathbf{c m}^{3}\right)\end{array}$ \\
\hline $\mathrm{B} 1$ & $77,96 \pm 0,52$ & $20,07 \pm 0,59$ & $29,67 \pm 0,58$ & $2,049 \pm 0,063$ & $0,488 \pm 0,015$ \\
\hline $\mathrm{B} 2$ & $80,61 \pm 0,14$ & $19,69 \pm 0,73$ & $27,33 \pm 0,29$ & $1,849 \pm 0,070$ & $0,541 \pm 0,020$ \\
\hline B3 & $81,52 \pm 0,30$ & $19,62 \pm 0,32$ & $29,00 \pm 0,87$ & $1,923 \pm 0,057$ & $0,520 \pm 0,015$ \\
\hline B4 & $82,49 \pm 0,79$ & $16,91 \pm 0,16$ & $26,67 \pm 0,38$ & $1,744 \pm 0,009$ & $0,573 \pm 0,003$ \\
\hline B5 & $81,72 \pm 0,45$ & $17,36 \pm 0,13$ & $26,33 \pm 0,58$ & $1,754 \pm 0,045$ & $0,570 \pm 0,015$ \\
\hline B6 & $81,34 \pm 0,25$ & $17,67 \pm 0,31$ & $26,67 \pm 0,38$ & $1,761 \pm 0,027$ & $0,568 \pm 0,009$ \\
\hline B7 & $81,64 \pm 0,10$ & $17,18 \pm 0,19$ & $26,83 \pm 0,76$ & $1,759 \pm 0,060$ & $0,569 \pm 0,020$ \\
\hline
\end{tabular}

EXP: Experimento.

O aumento da concentração de farinha de cascas de banana e de açúcar afetou negativamente o crescimento de bolos, em especial por causa da diluição do trigo e, consequentemente, da redução do conteúdo da proteína glúten, necessária para a expansão e o crescimento dos bolos durante o processamento (Soares et al., 2018), fenômeno semelhante reportado por Camargo et al. (2018), que constataram que a redução de proteína em massa de bolo de banana reduziu significativamente a altura em $25,69 \%$. Somado a isso, deve-se considerar que os bolos com maiores teores de fibras apresentavam massa mais pesada, dificultando a expansão do produto durante a cocção (Oliveira et al., 2016). Essas observações justificam as flutuações nas alturas, que oscilaram entre 16,91 mm na amostra processada com 15\% de farinha de cascas de banana e 15\% de açúcar (B4) e 20,07 mm no bolo feito com 5\% de farinha de cascas de banana e 5\% de açúcar (B1), aproximando-se da faixa relatada por Soares et al. (2018), que, ao estudarem o efeito da adição de proteína do soro do leite como substituto do trigo na formulação de bolos sem adição de açúcar, observaram alturas compreendidas entre 18,90 e $31,10 \mathrm{~mm}$.

Os volumes aparente e específico correlacionaram-se positivamente entre si, sendo mais elevados nas amostras processadas com as menores concentrações de farinha de cascas de banana (B1 e B3) e mais baixos em níveis nas maiores proporções desse resíduo agroindustrial. Esse fenômeno pode estar relacionado à viscosidade da massa, a qual aumentava à medida que se adicionava a farinha de cascas de banana, o que dificultou a areação dela durante a mistura, antes da cocção (Turker et al., 2016), indicando que danos à rede de glúten podem ter ocorrido (Silva et al., 2017) pela diluição dessa proteína, como já discutido. Soares et al. (2018) relataram que, quando a massa utilizada no processamento de bolos apresenta quantidade insuficiente de proteínas, especialmente o glúten, ocorre redução na retenção de gás nela, formando uma estrutura compacta e de baixo volume. Percebeu-se ainda que a adição de açúcar na massa também pode ter contribuído para a obtenção de volumes aparentes e específicos mais baixos, possivelmente relacionados à sua ligação com a água da massa, bem como a absorção de parte desse solvente pelas fibras da farinha.

De acordo com Souza et al. (2018), o vapor de água é considerado um agente de crescimento na massa, evaporando e expandindo a massa do bolo durante o cozimento. Logo, a provável redução da água livre pode ter contribuído para os menores volumes aparentes e específicos. Estudos desenvolvidos por Agrahar-Murugkar et al. (2018) e Silva et al. (2017) correlacionaram a redução dos volumes aparentes e/ou específicos de bolos com a incorporação de fibras presentes de farinhas agroindustriais, corroborando os resultados dos bolos deste trabalho.

As massas específicas apresentaram-se compreendidas entre 0,488 e $0,573 \mathrm{~g} \mathrm{~cm}^{-3}$, em que as adições de farinha de cascas de banana e de açúcar contribuíram para a obtenção de produtos mais densos. De acordo com Agrahar-Murugkar et al. (2018), bolos de maior qualidade estão associados à estrutura leve, porosa, alto 
volume e baixa massa específica (Agrahar-Murugkar et al., 2018), sendo observado que, em diferentes níveis de farinha de cascas de banana e de açúcar, a capacidade de reter e impedir a difusão do ar foi afetada. Isso pode estar relacionado à menor incorporação de ar durante a mistura da massa e às reduções proporcionais do glúten, de fermento químico e de gordura, comportamento relatado por Camargo et al. (2018) em bolos de banana, em que a massa específica reduziu em torno de $22 \%$ com a redução de proteínas na massa. Turker et al. (2016) estudaram o efeito da farinha de cascas de banana-verde nas características físicas de bolos e verificaram que, em concentrações elevadas de farinha, as amostras apresentaram maiores massas específicas. Zambrano et al. (2005) ainda afirmaram que misturas com menor conteúdo de gordura produzem bolos com massas específicas maiores, uma vez que esse ingrediente pode afetar as características de incorporação de ar. Apesar disso, muitos consumidores já associam as características de baixo volume e de maior massa específica a bolos com elevados teores de fibras.

Os resultados das análises de umidade, sólidos totais e SST dos bolos processados com diferentes concentrações de farinha da casca de banana e de açúcar estão apresentados na Tabela 3. Verificou-se que a umidade e os sólidos totais se apresentaram inversamente relacionados, com valores variando de $23,32 \%$ a $25,41 \%$ e de $74,59 \%$ e $76,68 \%$, respectivamente, sendo que o aumento da concentração de açúcares reduziu a umidade e elevou os sólidos totais, o que está relacionado à provável queda da água livre das massas pela adição do açúcar e à sua característica de elevado teor de sólidos (Bettani et al., 2014). Oliveira et al. (2016) informaram que a umidade é de grande relevância em bolos sob muitos aspectos, podendo contribuir para o aumento da atividade microbiana e consequente pegajosidade. Apesar disso, deve-se considerar que a umidade de bolos apresenta uma tendência à redução com a estocagem, fazendo com que as migalhas dos produtos assados se tornem secas e duras (Samsudin et al., 2019). No menor nível de açúcar (B1 e B2), observou-se que o aumento da concentração de farinha de cascas de banana aumentou a umidade em 5,65\%, associado à capacidade das fibras em absorver água (Jeddou et al., 2017) e reter a umidade durante o processo de cozimento (Agrahar-Murugkar et al., 2018). Em níveis mais elevados de açúcar, não foi possível observar esse efeito. A literatura apresenta diferentes comportamentos para a adição de farinhas agroindustriais como substitutas à farinha de trigo. Rosa et al. (2015) reportaram que a adição de farinha de alfarroba em bolos de banana contribuiu para o aumento da umidade, enquanto Silva et al. (2018) relataram que a incorporação de $20 \%$ de farinha residual de umbu-cajá reduziu a umidade em 13,28\% em cupcakes. Por outro lado, Sodchit et al. (2013) não verificaram diferença significativa nos teores de umidade em bolos processados com celulase de farinha de banana. Em relação aos SST, percebeu-se que o açúcar foi a variável independente que mais afetou os seus valores. Em um mesmo nível de farinha de farinha de cascas de banana (B1 e B3 ou B2 e B4), incrementos de açúcar aumentaram em torno de $36 \%$ os SST, o que era esperado, visto que esse adoçante apresenta conteúdos elevados de glicídios (Bettani et al., 2014).

Tabela 3. Resultados médios das análises de umidade, sólidos totais e sólidos solúveis totais (SST) dos bolos processados com diferentes concentrações de farinha de cascas de banana e de açúcar.

\begin{tabular}{cccc}
\hline EXP & Umidade (\%) & Sólidos totais (\%) & SST ('Brix) \\
\hline B1 & $24,05 \pm 0,04$ & $75,95 \pm 0,04$ & $16,58 \pm 0,29$ \\
\hline B2 & $25,41 \pm 0,09$ & $74,59 \pm 0,09$ & $16,92 \pm 0,17$ \\
\hline B3 & $23,85 \pm 0,15$ & $76,15 \pm 0,15$ & $22,67 \pm 0,36$ \\
\hline B4 & $23,32 \pm 0,12$ & $76,68 \pm 0,12$ & $23,00 \pm 0,23$ \\
\hline B5 & $24,43 \pm 0,26$ & $75,57 \pm 0,26$ & $20,20 \pm 0,18$ \\
\hline B6 & $24,28 \pm 0,27$ & $75,72 \pm 0,27$ & $20,02 \pm 0,03$ \\
\hline B7 & $24,87 \pm 0,16$ & $75,13 \pm 0,16$ & $20,15 \pm 0,11$ \\
\hline
\end{tabular}

EXP: Experimento. 
Na Tabela 4 constam os resultados das análises de ATT, pH e relação SST/ATT dos bolos processados com diferentes concentrações de farinha da casca de banana e de açúcar. O aumento das concentrações de farinha de cascas de banana promoveu elevação da ATT, provavelmente pela incorporação de ácidos orgânicos presentes nesse insumo. Em contrapartida, a redução dos níveis de açúcar promoveu redução da ATT em virtude da diluição dos ácidos orgânicos presentes na massa, visto que o açúcar é um adoçante de baixa acidez (Bettani et al., 2014). Os bolos deste trabalho tiveram ATT variando entre $0,454 \%$ e $0,752 \%$, apresentando-se superiores aos resultados de Gorgônio et al. (2011), que, ao elaborarem bolos com diferentes níveis de farinha de sementes de abóbora, reportaram aumento da ATT, com valores compreendidos entre $0,36 \%$ e $0,63 \%$. O pH mostrou-se inversamente relacionado à ATT, em que o aumento da concentração de farinha de cascas de banana reduziu esse parâmetro, enquanto o incremento de açúcar aumentou o pH. Apesar dessas diferenças, todos os bolos apresentaram valores de $\mathrm{pH}$ superiores a 6,0 , o que pode favorecer a deterioração microbiana. Como os bolos são produtos de umidade intermediária, Samsudin et al. (2019) relataram que as atividades de água nesses produtos podem variar entre 0,85 e 0,91 . Assim, ainda que os bolos deste trabalho tenham apresentado dados de $\mathrm{pH}$ elevados, a umidade intermediária pode favorecer crescimento de micro-organismos deteriorantes mais tolerantes em ambientes de umidades intermediárias, especialmente os bolores e as leveduras.

Quanto à relação SST/ATT, notou-se que níveis mais baixos de farinha de cascas de banana e mais altos de açúcar elevaram essa variável em até $134,15 \%$, obtendo-se valores de 22,49 a 52,66, sugerindo que esses bolos eram sensorialmente mais doces, visto que a percepção de doçura é função do melhor equilíbrio entre os ácidos orgânicos com os sólidos em solução do alimento. Gorgônio et al. (2011) encontraram em bolos isentos de açúcar, processados com diferentes concentrações de farinha de sementes de abóbora, valores para a relação SST/ATT compreendidos entre 2,98 e 11,05, inferiores aos deste estudo.

Tabela 4. Resultados médios das análises de acidez total titulável (ATT), pH e relação SST/ATT dos bolos processados com diferentes concentrações de farinha de cascas de banana e de açúcar.

\begin{tabular}{cccc}
\hline EXP & ATT (\%) & pH & Relação SST/ATT \\
\hline B1 & $0,454 \pm 0,024$ & $6,38 \pm 0,06$ & $36,49 \pm 0,37$ \\
\hline B2 & $0,752 \pm 0,013$ & $6,06 \pm 0,05$ & $22,49 \pm 0,28$ \\
\hline B3 & $0,430 \pm 0,008$ & $6,61 \pm 0,06$ & $52,66 \pm 0,31$ \\
\hline B4 & $0,609 \pm 0,033$ & $6,10 \pm 0,07$ & $37,79 \pm 0,44$ \\
\hline B5 & $0,580 \pm 0,016$ & $6,30 \pm 0,01$ & $34,84 \pm 0,19$ \\
\hline B6 & $0,568 \pm 0,012$ & $6,31 \pm 0,04$ & $35,26 \pm 0,17$ \\
\hline B7 & $0,574 \pm 0,009$ & $6,33 \pm 0,03$ & $35,09 \pm 0,21$ \\
\hline
\end{tabular}

EXP: Experimento; SST: sólidos solúveis totais.

Na Tabela 5 estão apresentadas as análises de regressão para os parâmetros físicos e químicos dos bolos processados com diferentes concentrações de farinha de cascas de banana e de açúcar. Cada parâmetro foi avaliado individualmente a partir do planejamento experimental fatorial, sendo gerados modelos matemáticos de $1^{\text {a }}$ ordem, com interação e intervalo de confiança de $95 \%$, além de valores de $\mathrm{F}$ calculado e de coeficientes de correlação $\left(\mathrm{R}^{2}\right)$ para cada variável dependente. Observou-se que apenas as respostas de SST, ATT, pH e relação SST/ATT apresentaram modelos matemáticos bem ajustados aos dados experimentais, com valores de $R^{2}$ superiores a 0,98 e relação $F_{\text {calculado }} / F_{\text {tabelado }}$ superior a 4,0 , indicando que esses parâmetros foram significativos e preditivos (Oliveira et al., 2014). De acordo com Silva et al. (2017), a análise dos modelos matemáticos permite correlacionar as variações das variáveis independentes (farinha de cascas de banana e de açúcar) com os parâmetros de qualidade dos bolos. Assim, percebeu-se efeito positivo da farinha de cascas de banana sobre os SST e a ATT e negativo sobre o pH e a relação SST/ATT. Em contrapartida, houve efeito 
positivo do açúcar sobre os SST, o pH e a relação SST/ATT e negativo sobre a ATT. Já a interação entre os as variáveis independentes revelaram efeito positivo sobre os SST e negativo sobre a ATT, o pH e a relação SST/ATT.

Tabela 5. Análises de regressão para os parâmetros físicos e químicos dos bolos processados com diferentes concentrações de farinha de cascas de banana e de açúcar.

\begin{tabular}{ccccc}
\hline Resposta & $\mathbf{R}^{2}$ & $\mathbf{F}_{\mathbf{c}}$ & $\mathbf{F}_{\mathbf{c}} / \mathbf{F}_{\text {tab }}$ & Modelo matemático \\
\hline Rendimento & 0,8804 & 7,3588 & 0,7932 & $81,0402+0,9033 \mathrm{FCB}+1,3577 \mathrm{~A}-0,4195 \mathrm{FCB} \mathrm{A}$ \\
\hline Altura & 0,5652 & 1,3000 & 0,1401 & $18,3562-0,7733 \mathrm{FCB}-0,8100 \mathrm{~A}-0,5817 \mathrm{FCB} \mathrm{A}$ \\
\hline Volume aparente & 0,5792 & 1,3766 & 0,1484 & $27,5000-1,1667 \mathrm{FCB}-0,3333 \mathrm{~A}+0,0000 \mathrm{FCB} \mathrm{A}$ \\
\hline Volume específico & 0,6176 & 1,6152 & 0,1741 & $1,83432-0,09456 \mathrm{FCB}-0,05777 \mathrm{~A}+0,00518 \mathrm{FCB} \mathrm{A}$ \\
\hline Massa específica & 0,6050 & 1,5314 & 0,1651 & $0,54691+0,02648 \mathrm{FCB}+0,01614 \mathrm{~A}+0,00016 \mathrm{FCB} \mathrm{A}$ \\
\hline Umidade & 0,8489 & 5,6188 & 0,6057 & $24,3168+0,2077 \mathrm{FCB}-0,5721 \mathrm{~A}-0,4736 \mathrm{FCB} \mathrm{A}$ \\
\hline Sólidos totais & 0,8489 & 5,6188 & 0,6057 & $75,6832-0,2077 \mathrm{FCB}+0,5721 \mathrm{~A}+0,4736 \mathrm{FCB} \mathrm{A}$ \\
\hline SST & 0,9945 & 180,8187 & 19,4911 & $19,9333+0,1667 \mathrm{FCB}+3,0417 \mathrm{~A}+0,00000 \mathrm{FCB} \mathrm{A}$ \\
\hline ATT & 0,9950 & 197,6885 & 21,3095 & $0,56676+0,11900 \mathrm{FCB}-0,04187 \mathrm{~A}-0,02988 \mathrm{FCB} \mathrm{A}$ \\
\hline pH & 0,9938 & 159,2539 & 17,1665 & $6,29771-0,20850 \mathrm{FCB}+0,06817 \mathrm{~A}-0,04483 \mathrm{FCB} \mathrm{A}$ \\
\hline Relação SST/ATT & 0,9804 & 50,1041 & 5,4009 & $36,3752-7,2194 \mathrm{FCB}+7,8659 \mathrm{~A}-0,2174 \mathrm{FCB} \mathrm{A}$ \\
\hline
\end{tabular}

$\mathrm{F}_{\text {tab }(3 ; 3 ; 0,5)}: 9,2770 ; \mathrm{F}_{\text {tab}}: \mathrm{F}$ tabelado; $\mathrm{F}_{\mathrm{c}}: \mathrm{F}$ calculado; $\mathrm{R}^{2}$ : Coeficiente de correlação; FCB: Farinha de cascas de banana; A: Açúcar; ATT: Acidez total titulável; SST: Sólidos solúveis totais.

Os diagramas de Pareto para os parâmetros de SST, ATT, pH e relação SST/ATT dos bolos processados com diferentes concentrações de farinha de cascas de banana e de açúcar estão dispostos na Figura 1. Percebeu-se que os SST sofreram influência significativa $(p<0,05)$ apenas do açúcar, enquanto o $\mathrm{pH}$, a ATT e a relação SST/ATT foram afetados significativamente $(p<0,05)$ tanto pelo açúcar quanto pela farinha de cascas de banana, sendo que a variável independente açúcar foi a que mais influenciou na relação SST/ATT, e a farinha de cascas de banana foi a variável independente que mais afetou a ATT e o pH. A interação entre os dois fatores independentes foi significativa $(p<0,05)$ apenas para ATT e $\mathrm{pH}$. 

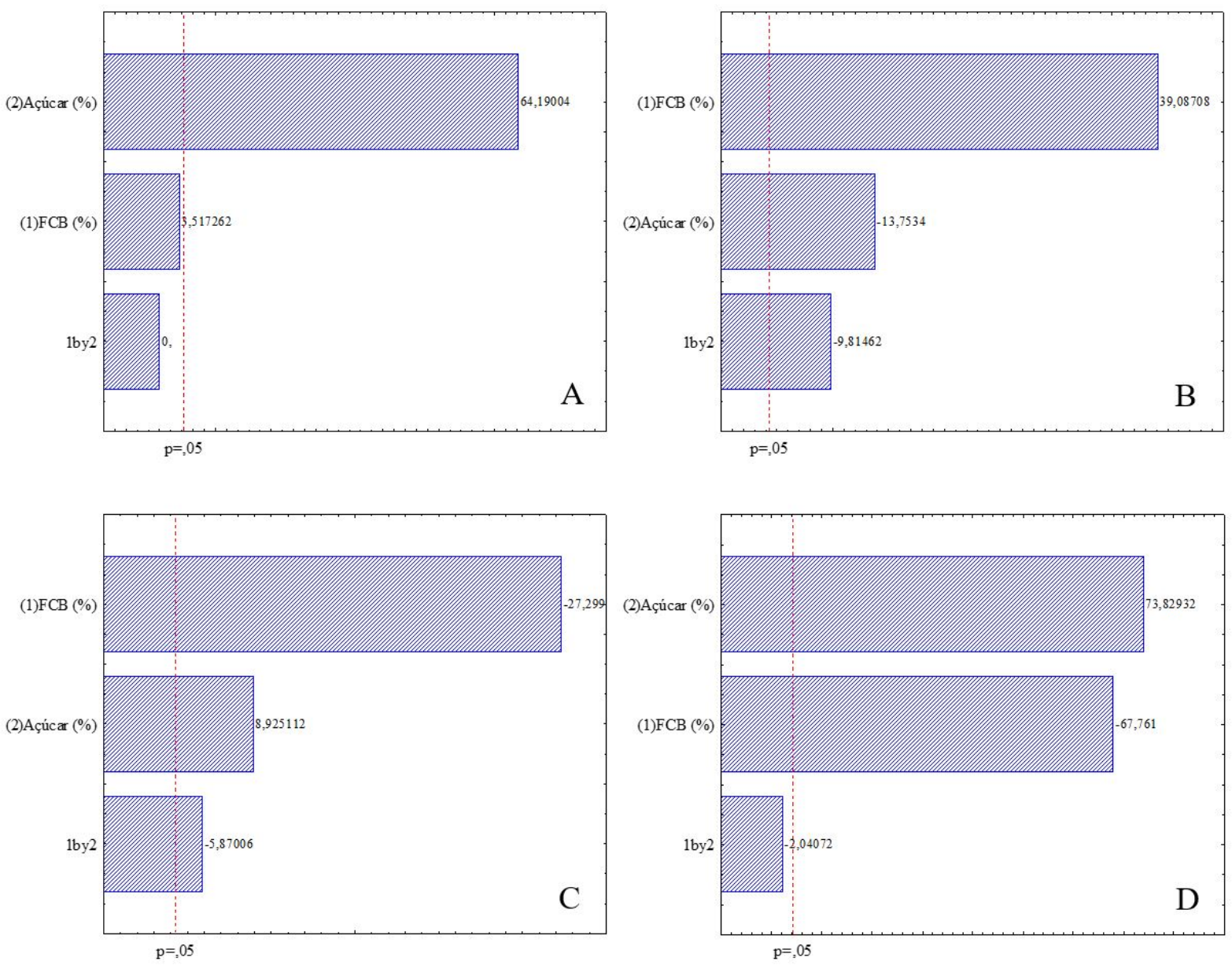

Figura 1. Diagramas de Pareto para os sólidos solúveis totais (A), acidez total titulável (B), $\mathrm{pH}$ (C) e relação SST/ATT (D) dos bolos processados com diferentes concentrações de farinha de cascas de banana (FCB) e de açúcar. 1by2: Interação entre o fator FCB (1) e o fator açúcar (2).

A partir dos diagramas de Pareto, verificou-se que pelo menos uma variável independente (ou sua interação) afetou significativamente $(p<0,05)$ os parâmetros de SST, ATT, pH e relação SST/ATT, portanto as superfícies de resposta puderam ser obtidas (Silva et al., 2017). Assim, a Figura 2 dispõe as superfícies de resposta para as variáveis dependentes significativas dos bolos processados com diferentes concentrações de farinha de cascas de banana e de açúcar. Observou-se que as superfícies de respostas se correlacionaram positivamente com os modelos de regressão e diagramas de Pareto, em que o aumento da concentração de farinha de cascas de banana aumentou a ATT e reduziu as respostas de pH e relação SST/ATT. Entretanto, elevando a proporção de açúcar, as respostas de SST e relação SST/ATT aumentaram consideravelmente, enquanto o pH e a ATT apresentaram pouca modificação, conforme já discutido (Tabela 4). 

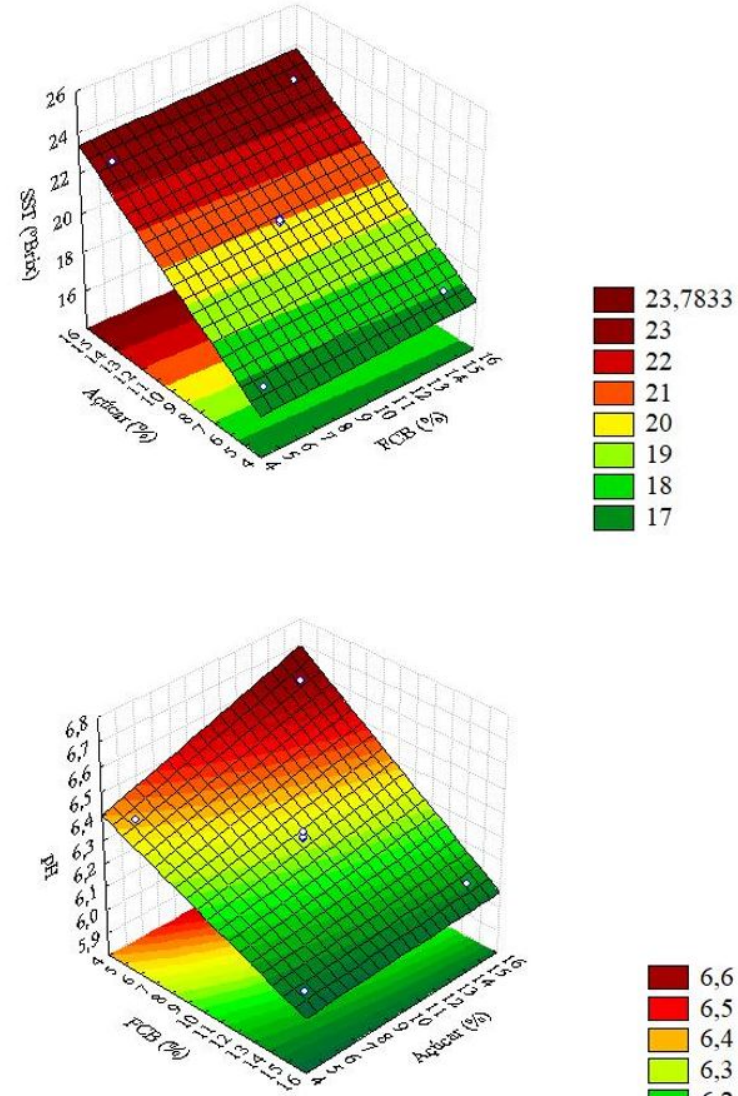
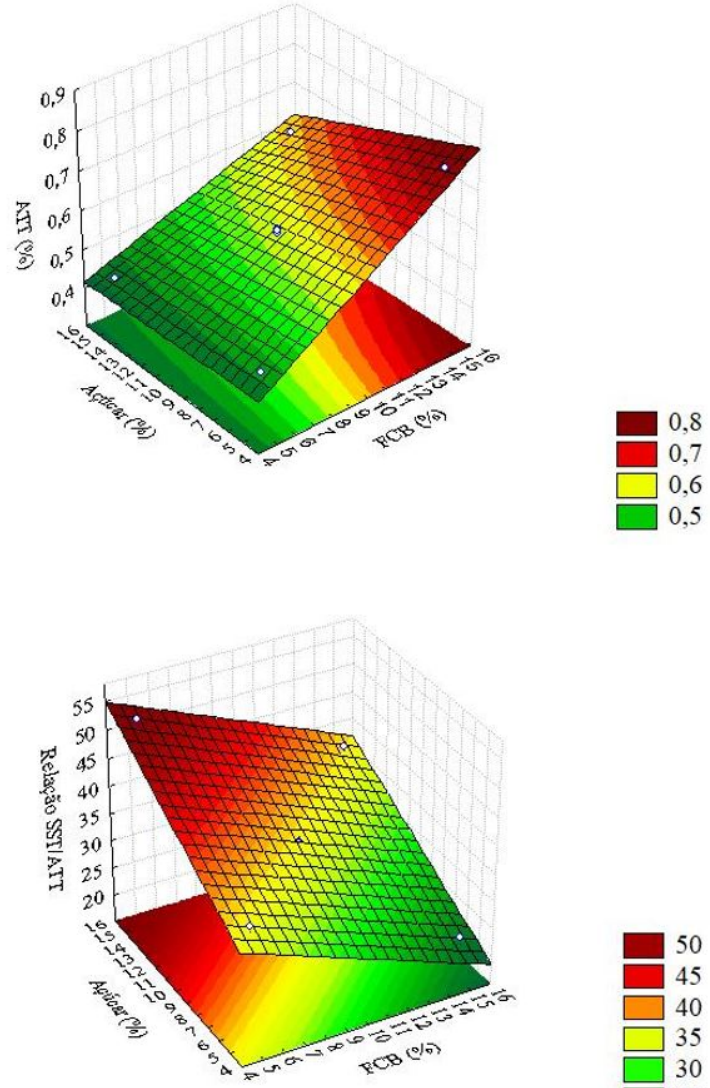

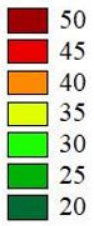

Figura 2. Superfícies de resposta para as variáveis dependentes de sólidos solúveis totais (SST), acidez total titulável (ATT), pH e relação SST/ATT dos bolos processados com diferentes concentrações de farinha de cascas de banana (FCB) e de açúcar.

\section{Conclusões}

Obtiveram-se bolos com variações de alguns atributos físicos e/ou químicos, sendo alguns deles característicos de bolos com elevado conteúdo e fibras e valor energético mais reduzido, porém é necessário haver estudos sensoriais e de textura instrumental para se estabelecer uma formulação otimizada. Os parâmetros de SST, ATT, pH e relação SST/ATT são significativos, em que o aumento da concentração de farinha de cascas de banana eleva a ATT e diminui o pH e a relação SST/ATT, enquanto incrementos de açúcar elevam os SST e a relação SST/ATT.

\section{Referências}

Agrahar-Murugkar, D., Zaidi, A., \& Dwivedi, S. (2018). Development of gluten free eggless cake using gluten free composite flours made from sprouted and malted ingredientes and its physical, nutritional, textural, rheological and sensory properties evaluation. Journal of Food Science and Technology, 55(7), 2621-2630. PMid:30042578. http://dx.doi.org/10.1007/s13197-0183183-1

Alves, E. J., Medina, V. M., \& Oliveira, M. (1999). Colheita e manejo pós-colheita. In E. J. Alves (Ed.), A cultura da banana: Aspectos técnicos, socioeconômicos e agroindustriais (2. ed). Cruz das Almas: EMBRAPA-CNPMF.

are Bettani, S. R., Lago, C. E., Faria, D. A. M., Borges, M. T. M. R., \& Verruma-Bernardi, M. R(2014). Avaliação físico-química e sensorial de açúcares orgânicos e convencionais. Revista Brasileira de Produtos Agroindustriais, 16(2), $155-162$. http://dx.doi.org/10.15871/1517-8595/rbpa.v16n2p155-162

Borges, C. V., Amorim, E. P., Leonel, M., Gomez, H. A. G., Santos, T. P. R., Ledo, C. A. S., Belin, M. A. F., Almeida, S. L., Minatel, I. O., \& Lima, G. P. P. (2019). Post-harvest physicochemical profile and bioactive compounds of 19 bananas and plantains genotypes. Bragantia, 78(2), 284-296. http://dx.doi.org/10.1590/1678-4499.20180252 
Camargo, L. R., Silva, L. M., Komeroski, M. R., Kist, T. B. L., Rodrigues, C. E., Rios, A. O., Silva, M. M., Doneda, D., Schmidt, H. O., \& Oliveira, V. R. (2018). Effect of whey protein addition on the nutritional, technological and sensory quality of banana cake. International Journal of Food Science \& Technology, 53(11), 2617-2623. http://dx.doi.org/10.1111/ijfs.13857

Carvalho, V. S., \& Conti-Silva, A. C. (2018). Cereal bars produced with banana peel flour: Evaluation of acceptability and sensory profile. Journal of the Science of Food and Agriculture, 98(1), 134-139. PMid:28543033.

http://dx.doi.org/10.1002/jsfa.8447

Castelo-Branco, V. N., Guimarães, J. N., Souza, L., Guedes, M. R., Silva, P. M., Ferrão, L. L., Miyahira, R. F., Guimarães, R. R., Freitas, S. M. L., Reis, M. C., \& Zago, L. (2017). The use of green banana (Musa balbisiana) pulp and peel flour as an ingredient for tagliatelle pasta. Brazilian Journal of Food Technology, 20(1), 1-8. http://dx.doi.org/10.1590/1981-6723.11916

Dias, C. S., Borges, S. V., Queiroz, F., \& Pereira, P. A. P. (2011). Influência da temperatura sobre as alterações físicas, físicoquímicas e químicas de geleia da casca de banana (Musa spp.) Cv. Prata durante o armazenamento. Revista do Instituto Adolfo Lutz, 70(1), 28-34.

Eshak, N. S. (2016). Sensory evaluation and nutritional value of balady flat bread supplemented with banana peels as a natural source of dietary fiber. Annals of Agricultural Science, 61(2), 229-235. http://dx.doi.org/10.1016/j.aoas.2016.07.002

Ghag, S. B., \& Ganapathi, T. R. (2019). Banana and plantains: Improvement, nutrition, and health. In J. M. Mérillon \& K. Ramawat (Eds.), Bioactive molecules in food (Reference Series in Phytochemistry, pp. 1755-1774). Cham: Springer. http://dx.doi.org/10.1007/978-3-319-78030-6_73.

Gorgônio, C. M. S., Pumar, M., \& Mothe, C. G. (2011). Macrocospic and physiochemical characterization of a sugarless and gluten-free cake enriched with fibers made from pumpkin seed (Cucurbita maxima, L.) flour and cornstarch. Food Science and Technology, 31(1), 109-118. http://dx.doi.org/10.1590/S0101-20612011000100015

Handique, J., Bora, S. J., \& Sit, N. (2019). Optimization of banana juice extraction using combination of enzymes. Journal of Food Science and Technology, 56(8), 3732-3743. PMid:31413400. http://dx.doi.org/10.1007/s13197-019-03845-z

Instituto Adolfo Lutz - IAL. (2008). Normas analíticas do Instituto Adolfo Lutz: Métodos físico-químicos para análises de alimentos (4. ed.). São Paulo: IAL.

Instituto Brasileiro de Geografia e Estatística - IBGE (2019). Produção agrícola municipal. Recuperado em 15 de julho de 2019, de https://sidra.ibge.gov.br/tabela/5457.

Jeddou, K. B., Bouaziz, F., Ellouzi, S. Z., Chaari, F., Chaabouni, S. E., Ghorbel, R. E., \& Ellouz, O. N. (2017). Improvement of texture and sensory properties of cakes by addition of potato peel powder with high level of dietary fiber and protein. Food Chemistry, 15(1), 668-677. PMid:27664685. http://dx.doi.org/10.1016/j.foodchem.2016.08.081

Khoozani, A. A., Birch, J., \& Bekhit, A. E. D. A. (2019). Production, application and health effects of banana pulp and peel flour in the food industry. Journal of Food Science and Technology, 56(2), 548-559. PMid:30906012. http://dx.doi.org/10.1007/s13197-018-03562-z

Oliveira, E. N. A., Rocha, A. P. T., Gomes, J. P., \& Santos, D. C. (2014). Influência das variáveis de processo nas características físicas e químicas de geleias de umbu-cajá. Bioscience Journal, 30(6), 1698-1710.

Oliveira, V. R., Preto, L. T., Schmidt, H. O., Komeroski, M., Silva, V. L., \& Rios, A. O. (2016). Physicochemical and sensory evaluation of cakes made with passion fruit and orange residues. Journal of Culinary Science \& Technology, 14(2), 166-175. http://dx.doi.org/10.1080/15428052.2015.1102787

Pizzinatto, A., \& Campagnolli, D. M. F. (1993). Avaliação tecnológica de produtos derivados de farinha de trigo (pão, macarrão, biscoito). Campinas: ITAL.

Rebello, L. P. G., Ramos, A. M., Pertuzatti, P. B., Barcia, M. T., Castillo-Muñoz, N., \& Hermosín-Gutiérrez, I. (2014). Flour of banana (Musa AAA) peel as a source of antioxidante phenolic compounds. Food Research International, 55(1), 397-403. http://dx.doi.org/10.1016/j.foodres.2013.11.039

Rocha, L. S., Santana, R. F., Soares, A. C. F., \& Haddad, F. (2018). Reaction of banana cultivars to the Meloidogyne javanica X Fusarium oxysporum f. sp. cubense complex. Revista Caatinga, 31(3), 572-583. http://dx.doi.org/10.1590/198321252018v31n305rc

Rosa, C. S., Tessele, K., Prestes, R. C., Silveira, M., \& Franco, F. (2015). Effect of substituting of cocoa powder for carob flour in cakes made with soy and banana flours. International Food Research Journal, 22(5), 2111-2118.

Sá, C. P., Nascimento, G. C., Nogueira, S. R., \& Andrade Neto, R. C. (2016). Coeficientes técnicos, custos de produção e indicadores econômicos para o cultivo da banana-comprida, cultivar D'Angola, no Acre (Comunicado Técnico, No. 191). Rio Branco: EMBRAPA Acre.

Samsudin, N. I. P., Roslan, N. A., Nor-Khaizura, M. A. R., \& Hasan, H. (2019). Shelf life extension of ambient-stored banana cake using banana powder. International Food Research Journal, 26(1), 305-312.

Silva, D., Pagani, A., \& Souza, R. (2018). Elaboração de cupcake adicionado de farinha de resíduo de umbu cajá: Características sensoriais e químicas. Revista Ciência (In) Cena, 1(7), 28-46.

Silva, M. L., Mendonça, L. G., Zambelli, R. A., Magalhães, A. L. M., Costa, C. S., Leão, M. V. S., \& Costa, R. A. R. S. (2017). Impact of green pulp banana and flaxseed flour on pound cake quality. International Journal of Nutrition and Food Sciences, 6(6), 243-249. http://dx.doi.org/10.11648/j.ijnfs.20170606.15

Soares, J. P., Marques, G. A., Magalhães, C. S., Santos, A. B., José, J. F. B. S., Silva, D. A., \& Silva, E. M. M. (2018). Efeito da adição de proteína do soro do leite como substituto do trigo na formulação de bolos sem adição de açúcar. Brazilian Journal of Food Technology, 21(1), 1-9. http://dx.doi.org/10.1590/1981-6723.19016 
Sodchit, C., Tochampa, W., Kongbangkerd, T., \& Singanusong, R. (2013). Effect of banana peel cellulose as a dietary fiber supplement on baking and sensory qualities of butter cake. Songklanakarin Journal of Science and Technology, 35(6), 641-646.

Souza, N. C. O., Oliveira, L. L., Alencar, E. R., Moreira, G. P., Leandro, E. S., Ginani, V. C., \& Zandonadi, R. P. (2018). Textural, physical and sensory impacts of the use of green banana puree to replace fat in reduced sugar pound cakes. Lebensmittel-Wissenschaft + Technologie, 89(1), 617-623. http://dx.doi.org/10.1016/j.Iwt.2017.11.050

Turker, B., Savlak, N., \& Kasikci, M. B. (2016). Effect of green banana peel flour substitution onphysical characteristics of gluten-free cakes. Current Research in Nutrition and Food Science, 4, 197-204. http://dx.doi.org/10.12944/CRNFSJ.4.SpecialIssue-October.25

Vu, H. T., Scarlett, C. J., \& Vuong, Q. V. (2018). Phenolic compounds within banana peel and their potential uses: A review. Journal of Functional Foods, 40(1), 238-248. http://dx.doi.org/10.1016/j.jff.2017.11.006

Zambrano, F., Hikage, A., Ormenese, R. C. C., Montenegro, F. M., \& Rauen-Miguel, A. M. (2005). Efeito das gomas guar e xantana em bolos como substitutos de gordura. Brazilian Journal of Food Technology, 8(1), 63-71.

Zhang, J., Jha, S. K., Liu, C., \& Hayashi, K. (2019). Tracing of chemical components of odor in peels and flesh from ripe banana on a daily basis using GC-MS characterization and statistical analysis for quality monitoring during storage. Food Analytical Methods, 12(4), 947-955. http://dx.doi.org/10.1007/s12161-019-01435-5 